\title{
Determinação da capacidade de acesso anterior à coluna cervicotorácica conforme idade e gênero: Análise radiográfica de imagens de tomografia computadorizada
}

\section{Determining Accessibility of Anterior Cervicothoracic Spine Based on Age and Gender: Radiographic Analysis of Computed Tomography Scans}

\author{
Rafael Lindi Sugino ${ }^{1,2}$ Alexandre Sadao lutaka1,2@ Samuel K. Cho ${ }^{3 \oplus}$ Daniel R. Cataldo ${ }^{30}$
} Luis Marchi ${ }^{1}$ Carlos Fernando Pereira da Silva Herrero ${ }^{40}$

\footnotetext{
${ }^{1}$ Divisão de Cirurgia de Coluna, Instituto Vita, São Paulo, SP, Brasil

2 Divisão de Cirurgia de Coluna, Hospital Israelita Albert Einstein, São Paulo, SP, Brasil

${ }^{3}$ Departamento de Cirurgia Ortopédica, Icahn School of Medicine at Mount Sinai, Nova York, NY, Estados Unidos

${ }^{4}$ Departamento de Ortopedia, Faculdade de Medicina de Ribeirão

Preto - Universidade de São Paulo (USP), Ribeirão Preto, SP, Brasil
}

Endereço para correspondência Rafael Lindi Sugino, MD, Rua Mato Grosso, 306, $1^{\circ}$ andar, São Paulo, SP, 01239-040, Brasil (e-mail: rsugino@gmail.com).

Rev Bras Ortop 2022;57(1):61-68.

\section{Resumo \\ Palavras-chave \\ - doenças da coluna vertebral \\ - fusão vertebral \\ - vértebras torácicas \\ - tomografia computadorizada por raios $\mathrm{X}$ \\ Objetivo A capacidade de acesso anterior à região cervicotorácica com base na idade e gênero do paciente e a possibilidade de variações anatômicas em diferentes populações geográficas ainda não foram investigadas. O objetivo deste trabalho foi realizar uma análise radiográfica de pacientes brasileiros para avaliar a acessibilidade anterior da junção cervicotorácica conforme idade e gênero. \\ Métodos Análise radiográfica retrospectiva de 300 tomografias computadorizadas. Os pacientes foram separados por idade e gênero. Os parâmetros radiográficos estudados foram: nível horizontal acima do esterno (HLS, na sigla em inglês), angulação do corpo vertebral (VBA, na sigla em inglês), linha do disco intervertebral (IDL, na sigla em inglês) e angulação da linha do disco intervertebral (IDLA, na sigla em inglês). \\ Resultados Os HLS e IDL mais frequentes foram T2 (34,3\%) e C7-T1 (46\%), respecti- vamente. Os valores médios de VBA e IDLA foram de $18 \pm 8,94$ e $19 \pm 7,9$ graus,}

Estudo realizado no Hospital das Clínicas de Ribeirão Preto (HCRP-USP), Ribeirão Preto, SP, Brasil.

recebido

30 de Junho de 2021

aceito

13 de Agosto de 2021

Publicado on-line

de Janeiro 21, 2022
DOI https://doi.org/

$10.1055 / \mathrm{s}-0041-1740295$.

ISSN 0102-3616. (c) 2022. Sociedade Brasileira de Ortopedia e Traumatologia. All rights reserved.

This is an open access article published by Thieme under the terms of the Creative Commons Attribution-NonDerivative-NonCommercial-License, permitting copying and reproduction so long as the original work is given appropriate credit. Contents may not be used for commercial purposes, or adapted, remixed, transformed or built upon. (https://creativecommons.org/ licenses/by-nc-nd/4.0/)

Thieme Revinter Publicações Ltda., Rua do Matoso 170, Rio de Janeiro, RJ, CEP 20270-135, Brazil 
respectivamente. Os homens apresentaram valores maiores de IDLA $(p=0,003)$ e VBA $(p=0,02)$. Os grupos de maior idade apresentaram valores maiores de IDLA $(p=0,01)$ e VBA $(p=0,001)$. Não houve diferenças de HLS entre os gêneros masculino e feminino $(p=0,3)$ ou faixas etárias $(p=0,79)$. Não foram observadas diferenças na IDL entre os gêneros masculino e feminino $(p=0,3)$; entretanto, o grupo mais velho apresentou nível mais caudal do que os grupos mais jovens $(p=0,12)$.

Conclusões Em comparação a outras populações, nossa amostra apresentou IDL e HLS mais cefálicos. AVBA e a IDLA foram maiores no gênero masculino, enquanto VBA e IDLA foram maiores em grupos mais velhos. A IDL era mais caudal em pacientes idosos.

\section{Abstract}

\section{Keywords}

- spinal diseases

- spinal fusion

- thoracic vertebrae

- tomography, x-ray computed
Objective The approachability of the cervicothoracic region anteriorly based on age and gender, and the possibility of anatomic variances in different geographic populations have not been previously investigated. The aim of the present work was to perform a radiographic analysis of Brazilian patients to assess anterior approachability of the cervicothoracic junction based on age and gender.

Methods Retrospective radiographic analysis of 300 computed tomography scans. Patients were separated based on age and gender. The radiographic parameters studied were: horizontal level above the sternum (HLS), vertebral body angle (VBA), intervertebral disc line (IDL), and intervertebral disc line angulation (IDLA).

Results The most frequent HLS and IDL were T2 (34.3\%) and C7-T1 (46\%) respectively. Vertebral body angleand IDLA had average values of $18 \pm 8.94$ and $19 \pm 7.9$ degrees, respectively. Males had higher values in both IDLA $(p=0.003)$ and VBA $(p=0.02)$. Older groups had higher values in both IDLA $(p=0.01)$ and VBA $(p=0.001)$. No differences were observed in HLS between gender $(p=0.3)$ or age groups $(p=0.79)$. No differences were seen in IDL between gender groups $(p=0.3)$; however, the older group had a more caudal level than the younger groups $(p=0.12)$.

Conclusions Compared to other populations, our sample had a more cephalad IDL and HLS. Vertebral body angle and IDLA were higher in males and higher angles for VBA and IDLA were shown for older groups. Intervertebral disc line was more caudal with aging.

\section{Introdução}

Em cirurgias de coluna, a junção cervicotorácica pode ser uma região de difícil acesso anterior. ${ }^{1}$ A abordagem mais comum é a anterolateral (Smith-Robinson), que permite 0 tratamento de níveis caudais, como T2. ${ }^{2-4}$ Dependendo da relação da coluna vertebral com o esterno e as clavículas, esta abordagem pode expor bem os níveis inferiores desejados sem necessidade de osteotomia do esterno ou das clavículas. $^{5-7}$

Muitas técnicas, inclusive transesternal, transclavicular e transmanubrial, foram descritas para superar as barreiras ósseas na abordagem da região cervicotorácica. No entanto, essas osteotomias são associadas a maiores taxas de morbidade e mortalidade. ${ }^{8-12}$ Por isso, medidas e parâmetros préoperatórios têm sido estudados para predizer a necessidade de realização dessas osteotomias durante a abordagem anterior à região cervicotorácica. Estudos anteriores utilizaram tomografia computadorizada (TC) ou ressonância magnética (RM) da coluna ${ }^{3,5,6,13}$ e diversos métodos de planejamento cirúrgico, desde uma simples linha de disco passando acima do esterno até trigonometria avançada com pontos anatômicos de referência.

A capacidade de acesso anterior à região cervicotorácica com base na idade e gênero do paciente ainda não foi investigada. Além disso, não há literatura que analise as possíveis variações anatômicas em diferentes populações geográficas. $\mathrm{O}$ objetivo deste estudo foi realizar uma análise radiográfica das imagens de TC de pacientes brasileiros para avaliar a capacidade de acesso anterior à junção cervicotorácica com base na idade e gênero nesta coorte.

\section{Materiais e Métodos}

Este foi um estudo radiográfico realizado em um único centro com aprovação do conselho de ética do hospital. Selecionamos tomografias realizadas entre 2008 e 2014. Os critérios de inclusão foram pacientes maiores de 18 anos com resultados normais no exame tomográfico. Os critérios de exclusão foram pacientes com tumor primário, metástases, infecção, cirurgia prévia da coluna vertebral, fraturas do esterno e/ou necessidade de procedimentos no esterno. Os 
Tabela 1 Parâmetros medidos na reconstrução sagital das imagens de tomografia computadorizada

\begin{tabular}{|l|l|l|}
\hline Parâmetro & Abreviatura & Descrição \\
\hline $\begin{array}{l}\text { Linha do disco } \\
\text { intervertebral }\end{array}$ & IDL & $\begin{array}{l}\text { Última IDL cau- } \\
\text { dal a passar } \\
\text { acima da fúrcula } \\
\text { esternal }\end{array}$ \\
\hline $\begin{array}{l}\text { Nível horizontal } \\
\text { acima do esterno }\end{array}$ & HLS & $\begin{array}{l}\text { Corpo vertebral } \\
\text { intersectado } \\
\text { por uma linha } \\
\text { horizontal a } \\
\text { partir da fúrcula } \\
\text { esternal }\end{array}$ \\
\hline $\begin{array}{l}\text { Angulação do corpo } \\
\text { vertebral }\end{array}$ & VBA & $\begin{array}{l}\text { Angulação do } \\
\text { corpo vertebral } \\
\text { intersectada por } \\
\text { HLS em uma } \\
\text { linha vertical ao } \\
\text { paciente }\end{array}$ \\
\hline $\begin{array}{l}\text { Angulação da linha do } \\
\text { disco intervertebral }\end{array}$ & IDLA & $\begin{array}{l}\text { Angulação da } \\
\text { IDA com uma } \\
\text { linha horizontal } \\
\text { ao paciente }\end{array}$ \\
\hline
\end{tabular}

Abreviaturas: HLS, nível horizontal acima do esterno; IDL, linha do disco intervertebral; IDLA, angulação da linha do disco intervertebral; VBA, angulação do corpo vertebral.

pacientes foram separados por gênero (masculino e feminino) e subdivididos por faixa etária (grupo 1: 18-40 anos; grupo 2: 41-60 anos e grupo 3: mais de 60 anos), criando seis grupos de 50 exames cada, com um total de 300 exames de TC. Gênero e idade foram os dois parâmetros clínicos adquiridos de prontuários médicos.

Todos os exames de TC foram realizados em decúbito dorsal horizontal com equipamento multislice Brilliance CT scanner Big Bore (Koninklijke Philips N.V., Amsterdã, Holanda) de 64 canais com cortes de $2 \mathrm{~mm}$. Todos os exames foram revistos pelo autor sênior, Dr. Rafael Lindi Sugino, com o software OsiriX MD (Pixmeo SARL, Bernex, Suíça), com aprovação da Food and Drug Administration (FDA), que permite reconstruções 3D em todos os planos, e é classificado como CE IIa. Todas as imagens foram reconstruídas em todos os três planos e alinhadas em todos os planos para medição.

\section{Avaliação Radiográfica}

Os seguintes parâmetros radiológicos foram estudados: 1) nível horizontal acima do esterno (HLS, na sigla em inglês); 2 ) angulação do corpo vertebral (VBA, na sigla em inglês); 3) linha do disco intervertebral (IDL) e 4) angulação da linha do disco intervertebral (IDLA, na sigla em inglês) ( - Tabela 1).

O HLS é o corpo vertebral seccionado por uma linha horizontal a partir da fúrcula esternal. Caso a linha cruzasse um disco vertebral, o corpo vertebral considerado era sempre o mais caudal ${ }^{5}$ (- Figura 1 ).

A VBA foi medida após a determinação do HLS. 0 ângulo entre uma linha traçada na face anterior do corpo vertebral

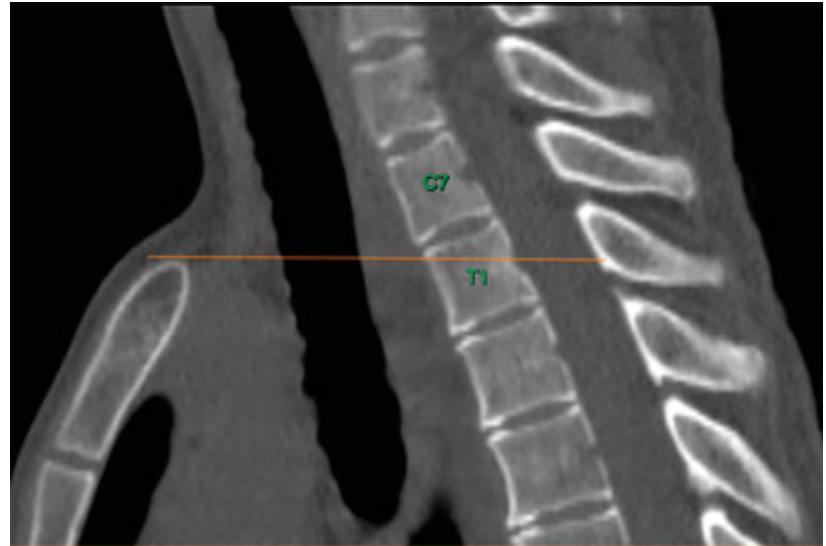

Fig. 1 Nível horizontal acima do esterno (HLS).

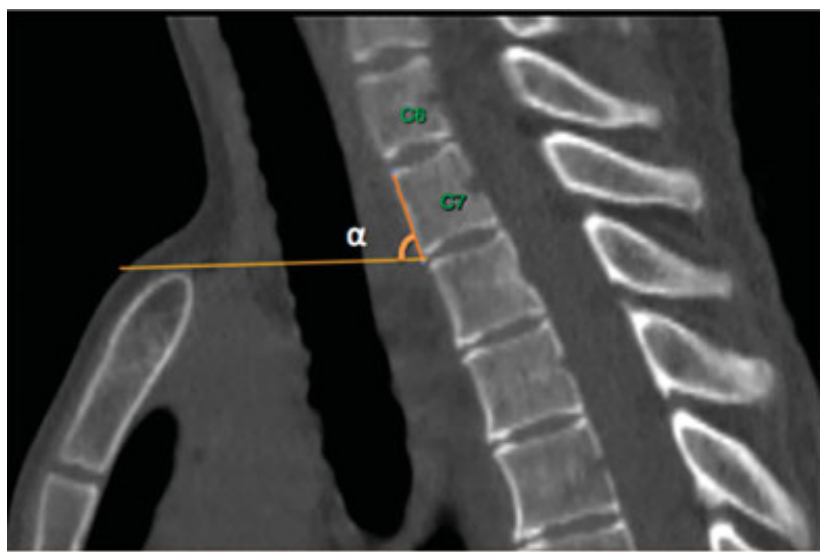

Fig. 2 Angulação do corpo vertebral (VBA) representada por $\alpha$.

e uma linha horizontal a partir da fúrcula esternal foi determinado. Este ângulo representa a rotação vertebral se HLS fosse escolhido como método pré-operatório (-Figura 2).

A IDL é definida pela linha do disco intervertebral mais caudal que passa acima da fúrcula esternal ${ }^{6}$ ( - Figura 3 ).

Depois da definição de IDL, o ângulo medido entre a IDL e uma linha horizontal representa a IDLA. Esta medida

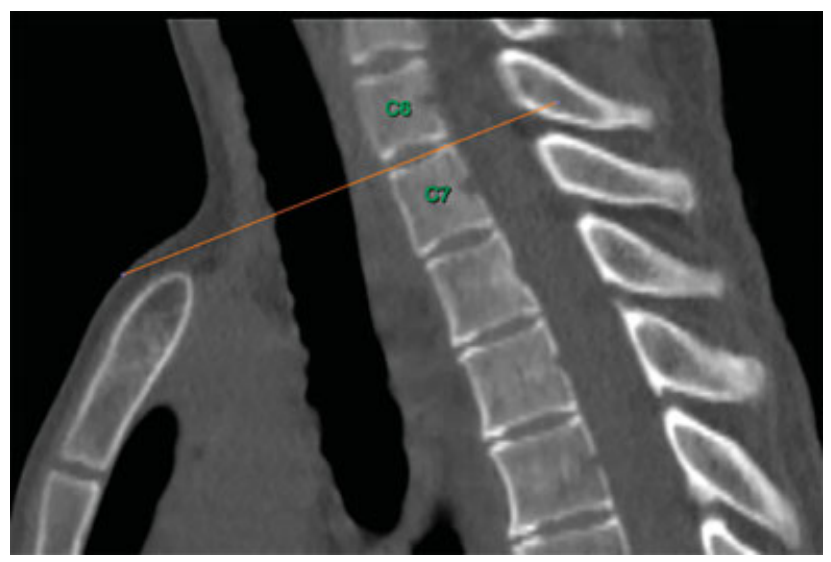

Fig. 3 Linha do disco intervertebral (IDL). 


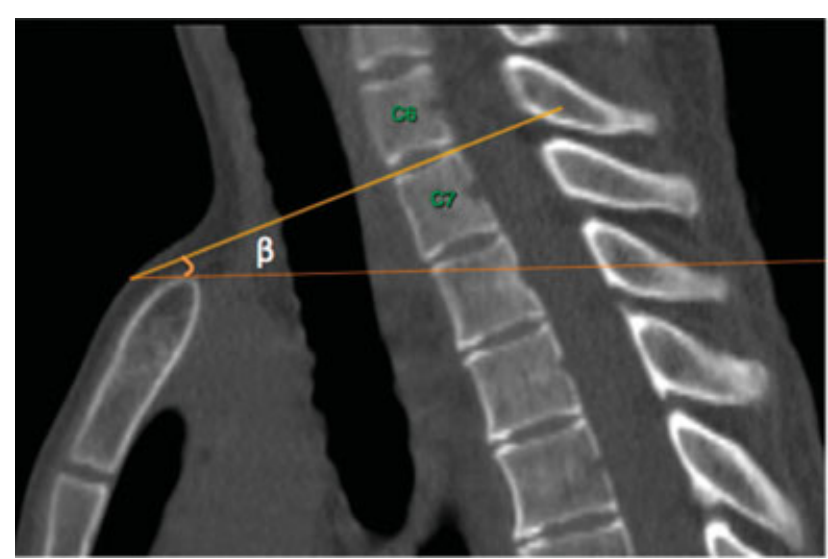

Fig. 4 Angulação do disco intervertebral (IDLA).

também pode ser interpretada como um ângulo de inclinação (-Figura 4).

\section{Análise Estatística}

As variáveis paramétricas IDLA e VBA são apresentadas como média e valores de desvio-padrão, enquanto as variáveis não paramétricas, IDL e HLS, são apresentadas como porcentagens.

Para fins deste estudo, os dados clínicos (idade e gênero) e variáveis não paramétricas (IDL e HLS) foram convertidos em variáveis ordinais para análise estatística.

As variáveis paramétricas foram submetidas ao teste de Shapiro-Wilke para assegurar a distribuição normal dos dados. Histogramas, assim como comparações de médias e medianas, foram utilizados com a mesma finalidade. Depois de determinar a normalidade da distribuição, os testes-t de Student e análise de variância (ANOVA) bidirecional foram realizados para comparação dos dados clínicos (idade e gênero); o pósteste de Bonferroni foi usado em análises posteriores.

Os dados não paramétricos IDL e HLS foram convertidos em valores ordinais para fins estatísticos. Como o nível C5-6 foi o nível mais cranial de IDL encontrado em nosso estudo, foi designado como número 1 , enquanto os níveis caudais subsequentes receberam valores ordinais sucessivos. 0 mesmo método foi utilizado para HLS. O nível mais cranial era o $\mathrm{C}$, que foi designado como número 1 .

As análises estatísticas de HLS e IDL foram baseadas no teste de qui-quadrado de Pearson. Todos os dados foram analisados com o software de análise estatística STATA13 (StataCorp. LLC. College Station, TX, EUA). A hipótese de nulidade de ausência de diferença foi rejeitada caso o valor de $p$ fosse inferior a 0,05 .

\section{Dados de Outros Estudos}

Em nossa pesquisa bibliográfica, coletamos dados análogos ao nosso delineamento experimental de outros artigos científicos. Todos os dados foram organizados para posterior comparação a nossos achados.

\section{Resultados}

No total, 300 participantes foram escolhidos. A média de idade foi de $50 \pm 23$ anos. A idade média do subgrupo masculino era de $49+/-18$ anos (intervalo: 18-91) e, do subgrupo feminino, era de $51+/-23$ (intervalo: 19-92).

O HLS variou de C7 a T4; T2 foi o nível mais frequente (34,3\%), seguido por T1-2 (20,7\%) e T2-3 (17,3\%). Os níveis menos comuns de HLS foram T4 (0,3\%) e C7 (1,0\%) (-Figura 5).

A VBA média foi de $18+/-8,94$ (intervalo: $1-54$ graus). A IDLA média foi de 19 graus +/- 7,9 (intervalo: 0-49 graus).

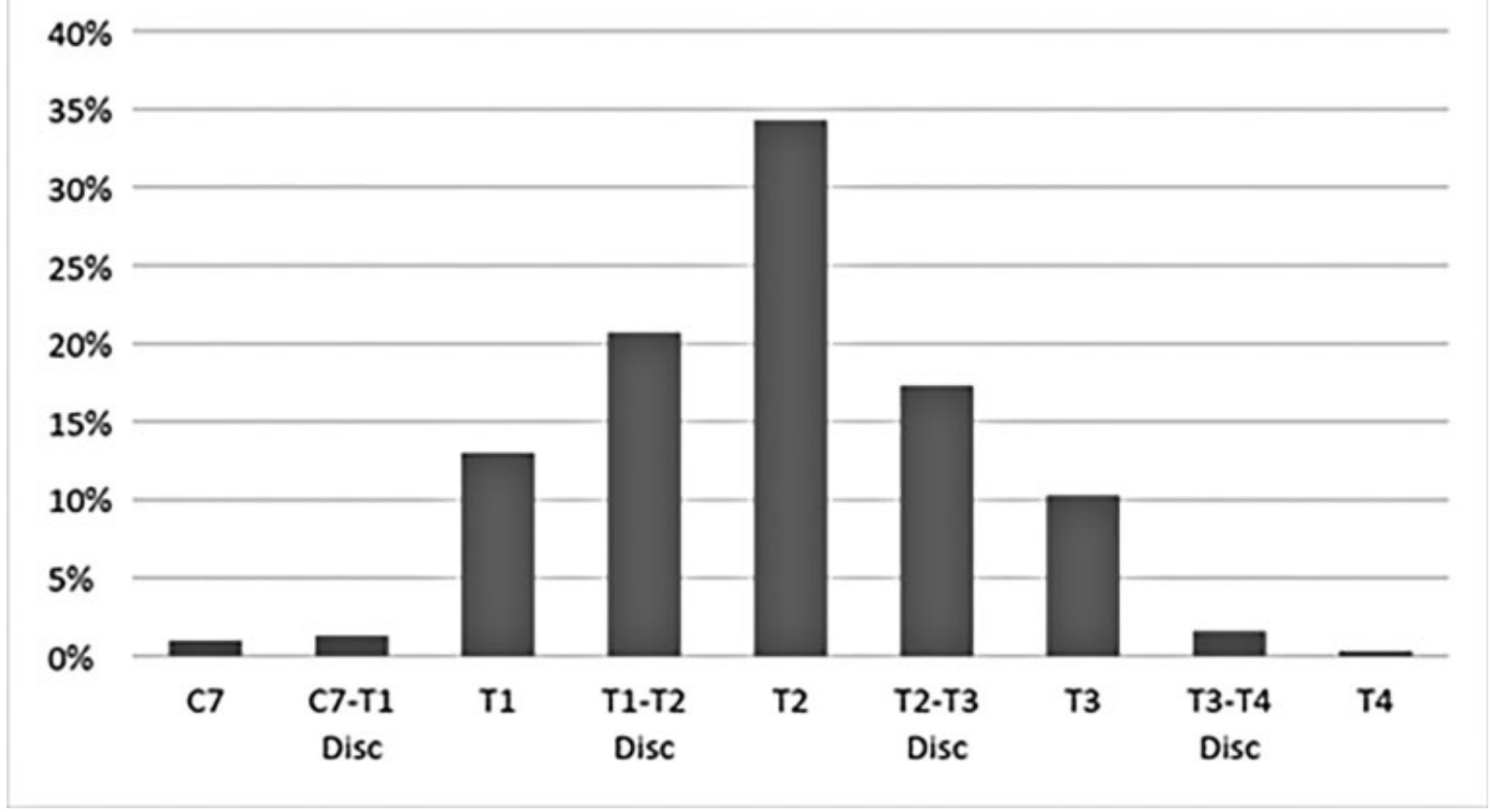

Fig. 5 Distribuição do nível horizontal acima do esterno (HLS) por nível no atual estudo. 


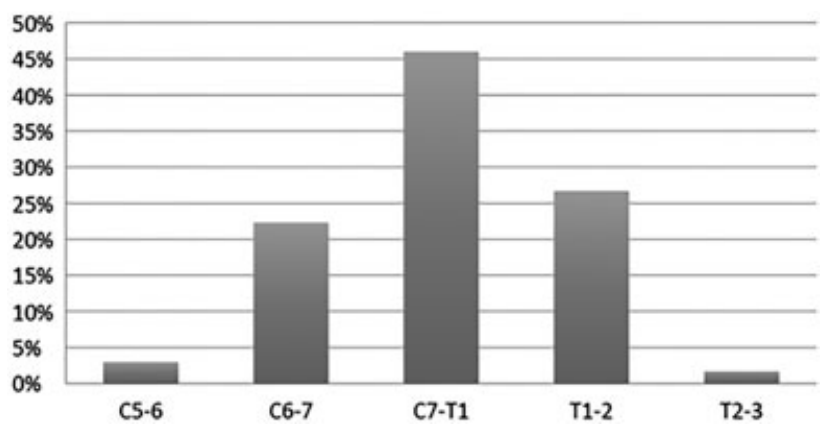

Fig. 6 Distribuição da linha do disco intervertebral (IDL) por nível no atual estudo.

Tabela 2 Valores-p de angulação da linha do disco intervertebral e angulação do corpo vertebral de acordo com idade e gênero

\begin{tabular}{|l|l|l|l|l|}
\hline & $\begin{array}{l}\text { Grupo 1 } \\
\text { vs 2 }\end{array}$ & $\begin{array}{l}\text { Grupo 2 } \\
\text { vs 3 }\end{array}$ & $\begin{array}{l}\text { Grupo 1 } \\
\text { vs 3 }\end{array}$ & Gênero \\
\hline IDLA & 0,001 & 0,01 & $<0,001$ & 0,03 \\
\hline VBA & 0,005 & $<0,001$ & 0,006 & 0,02 \\
\hline
\end{tabular}

Abreviaturas: IDLA, angulação da linha do disco intervertebral; VBA, angulação do corpo vertebral.

A IDL variou de C5-6 a T2-3; o nível mais frequente foi C7T1 (46\%), seguido pelos níveis T1-T2 (26,7\%) e C6-7 (22,3\%). Os níveis menos comuns de IDL foram T2-T3 $(1,7 \%)$ e C5-6 (3\%) (-Figura 6).

Quando comparando os grupos masculino e feminino, encontramos diferenças estatisticamente significativas tanto em IDLA $(p=0,003)$ quanto VBA $(p=0,02)$; os valores foram maiores em homens. Em relação à idade, diferenças estatisticamente significativas foram encontradas entre todas as faixas etárias tanto em IDLA $(p=0,01)$ quanto em VBA $(p=0,001)$; os grupos mais velhos apresentaram valores maiores. Uma análise posterior com o pós-teste de Bonferroni revelou diferenças estatisticamente significativas de IDLA e VBA entre todas as combinações possíveis das três faixas etárias; os valores-p são mostrados na - Tabela 2.

Não houve diferenças estatisticamente significativas em HLS entre homens e mulheres ( $p=0,3$ ) ou as diferentes faixas etárias $(p=0,79)$ ( - Tabela 3 ). Não houve diferença em IDL entre homens e mulheres $(p=0,4)$. No entanto, houve uma diferença entre as faixas etárias $(p<0,001)$, com nível mais caudal no grupo de maior idade ( - Tabela $\mathbf{3}$ ).

Tabela 3 Valores de $p$ de linha do disco intervertebral e nível horizontal acima do esterno de acordo com idade e gênero

\begin{tabular}{|l|l|l|}
\hline & Idade & Gênero \\
\hline HLS & $p=0,395$ & $p=0,799$ \\
\hline IDL & $p<0,001$ & $p=0,4$ \\
\hline
\end{tabular}

Abreviaturas: HLS, nível horizontal acima do esterno; IDL, linha do disco intervertebral.
Tabela 4 Distribuição de linha do disco invertebral em diferentes estudos

\begin{tabular}{|l|l|l|l|l|}
\hline IDL & $\begin{array}{l}\text { Sugino } \\
\text { et al. }\end{array}$ & $\begin{array}{l}\text { Sharan } \\
\text { et al. }\end{array}$ & $\begin{array}{l}\text { Karikari } \\
\text { et al. }\end{array}$ & $\begin{array}{l}\text { Mai } \\
\text { et al. }\end{array}$ \\
\hline C5-C6 & $100 \%$ & $100 \%$ & $100 \%$ & $100 \%$ \\
\hline C6-C7 & $96,6 \%$ & $99,0 \%$ & $99,4 \%$ & $92,7 \%$ \\
\hline C7-T1 & $74,3 \%$ & $95,2 \%$ & $91,7 \%$ & $85,5 \%$ \\
\hline T1-T2 & $28,3 \%$ & $59,4 \%$ & $82,7 \%$ & $62,2 \%$ \\
\hline T2-T3 & $1,6 \%$ & $14,1 \%$ & $17,3 \%$ & $16,1 \%$ \\
\hline T3-T4 & $0,0 \%$ & $0,0 \%$ & $0,0 \%$ & $0,0 \%$ \\
\hline
\end{tabular}

Abreviatura: IDL, linha do disco intervertebral.

Em nossa amostra, IDL e HLS apresentaram valores mais cefálicos em comparação a outras amostras populacionais já relatadas. Os resultados dos dados anteriores de IDL e HLS são mostrados nas-Tabelas 4 e 5; a - Figura 7 mostra as referências e os valores dos parâmetros.

\section{Discussão}

O planejamento pré-operatório é muito importante na cirurgia da coluna vertebral. A falta de atenção nesta etapa, principalmente na junção cervicotorácica, pode produzir desfechos ruins e complicações evitáveis relacionadas ao procedimento cirúrgico. A decisão de realizar osteotomia na abordagem cervicotorácica anterior depende de vários fatores, como peso do paciente, pescoço curto, obesidade, alinhamento local da coluna e extensão da patologia.

Para tanto, em 2000, Sharan et al. ${ }^{14}$ estudaram uma linha que seria tangencial à fúrcula e uma linha que dividiria o disco intervertebral ao meio no plano sagital, denominada o "disco intervertebral mais inferior visualizado" pelos pesquisadores. Segundo esses autores, a osteotomia poderia ser evitada se ambas as linhas fossem caudais à patologia sendo tratada. ${ }^{14}$

Em 2002, Frazer et al. ${ }^{3}$ testaram novos parâmetros para auxiliar cirurgiões na abordagem à junção cervicotorácica anterior. No entanto, em nossa opinião, esse método usava trigonometria avançada, e é complexo demais para uso rotineiro.

Karikari et al., ${ }^{6}$ em 2009, usaram imagens de RM e testaram clinicamente o mesmo conceito de "disco intervertebral mais inferior visualizado" usado por Sharan et al. ${ }^{14}$ para prever quando uma osteotomia do esterno não seria necessária. No mesmo ano, Teng et al. ${ }^{15}$ propuseram um novo ângulo cervicotorácico. 0 ângulo é formado por uma linha horizontal a partir da fúrcula esternal e uma segunda linha da fúrcula até o espaço C7-T1. A osteotomia era considerada necessária dependendo de onde a patologia estava localizada neste ângulo.

Em 2011, Falavigna et al. ${ }^{13}$ descreveram uma "linha do cirurgião para o disco" semelhante à descrita por Sharan et al. ${ }^{14}$ como um método pré-operatório na junção cervicotorácica.

Esses estudos tentaram desenvolver métodos confiáveis e simples para melhor planejamento cirúrgico. Para nosso 
Tabela 5 Distribuição do nível horizontal acima do esterno em diferentes estudos

\begin{tabular}{|l|l|l|l|l|l|l|l|l|l|}
\hline & C7 & C7-T1 & T1 & T1-2 & T2 & T2-3 & T3 & T3-4 & T4 \\
\hline Sharan et al. & $0,94 \%$ & - & $1,89 \%$ & - & $41,51 \%$ & - & $49,06 \%$ & - & $6,60 \%$ \\
\hline Lakishmanam et al. & - & - & $3,66 \%$ & $2,44 \%$ & $19,51 \%$ & $31,71 \%$ & $34,15 \%$ & $4,88 \%$ & $3,66 \%$ \\
\hline Teng et al. & - & - & - & - & $26,32 \%$ & $20,00 \%$ & $42,11 \%$ & $10,53 \%$ & $1,05 \%$ \\
\hline Sugino et al. & $1,00 \%$ & $1,33 \%$ & $13,00 \%$ & $20,67 \%$ & $34,33 \%$ & $17,33 \%$ & $10,33 \%$ & $1,67 \%$ & $0,33 \%$ \\
\hline
\end{tabular}

estudo, escolhemos os dois métodos que acreditamos gerar as técnicas mais confiáveis, simples e reproduzíveis em termos de planejamento cirúrgico. Em seguida, surgiram duas dúvidas: a população brasileira tem a mesma anatomia das populações já estudadas e esses parâmetros sofrem algum tipo de variação conforme a faixa etária ou o gênero do paciente?

\section{Comparação de Diferentes Populações}

Após revisão da literatura, três estudos com análises radiográficas semelhantes foram utilizados para comparação. 0 nível mais comum de IDL em nosso estudo foi T2. Isso difere de estudos anteriores que relataram um IDL mais caudal (-Figura 7). Em nosso estudo, por exemplo, T2-3 foi a IDL em $1,6 \%$ do tempo, enquanto Sharan et al. relatou o valor como $14,1 \%{ }^{14}$ Karikari et al. ${ }^{6} 17,3 \%$ e Mai et al. ${ }^{16} 16,1 \%$. Ao considerar o nível T1-2, nosso estudo (28,3\%) apresentou diferença ainda maior em comparação a outras populações (59,4\%, 82,7\% e 62,2\%, respectivamente) (-Tabela 4).

Em termos de HLS, o nível mais comum em nosso estudo foi T2 (34,33\%), seguido por T1-T2 (20,67\%) e T2-3 (17,33\%) (-Tabela 5). Por outro lado, Lakshmanam et al. $(34,15 \%)^{7}$ e Teng et al. $(42,11 \%)^{15}$ relataram T3 como o nível mais comum de HLS, seguido por T2-T3 (31,71\%) e T2 (19,51\%) no estudo

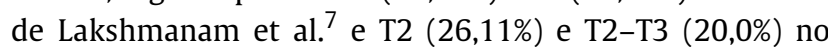
estudo de Teng et al. ${ }^{15}$ (-Figura 8).

Essas diferenças indicam, mais uma vez, que a população brasileira apresenta anatomia menos favorável para o tratamento da junção cervicotorácica, com possível necessidade de ultrapassar a anatomia óssea durante a abordagem. Em outras palavras, o tratamento de patologias anteriores seria mais difícil na população brasileira.

\section{Níveis e Dados Demográficos}

Na comparação entre grupos de diferentes idades e gêneros, o HLS não apresentou diferença estatística. Essa medida também pode representar a translação vertical indireta da coluna em relação ao esterno. 0 envelhecimento provoca um aumento normal da cifose torácica, o que pode alterar o HLS. No entanto, em nosso estudo, não vimos nenhuma diferença translacional com o envelhecimento.

Quando comparando grupos de diferentes gêneros e idades, a VBA e a IDLA tiveram comportamento semelhante. Esse achado pode ser explicado porque, à trigonometria, VBA e IDLA representam a mesma medida em níveis adjacentes,

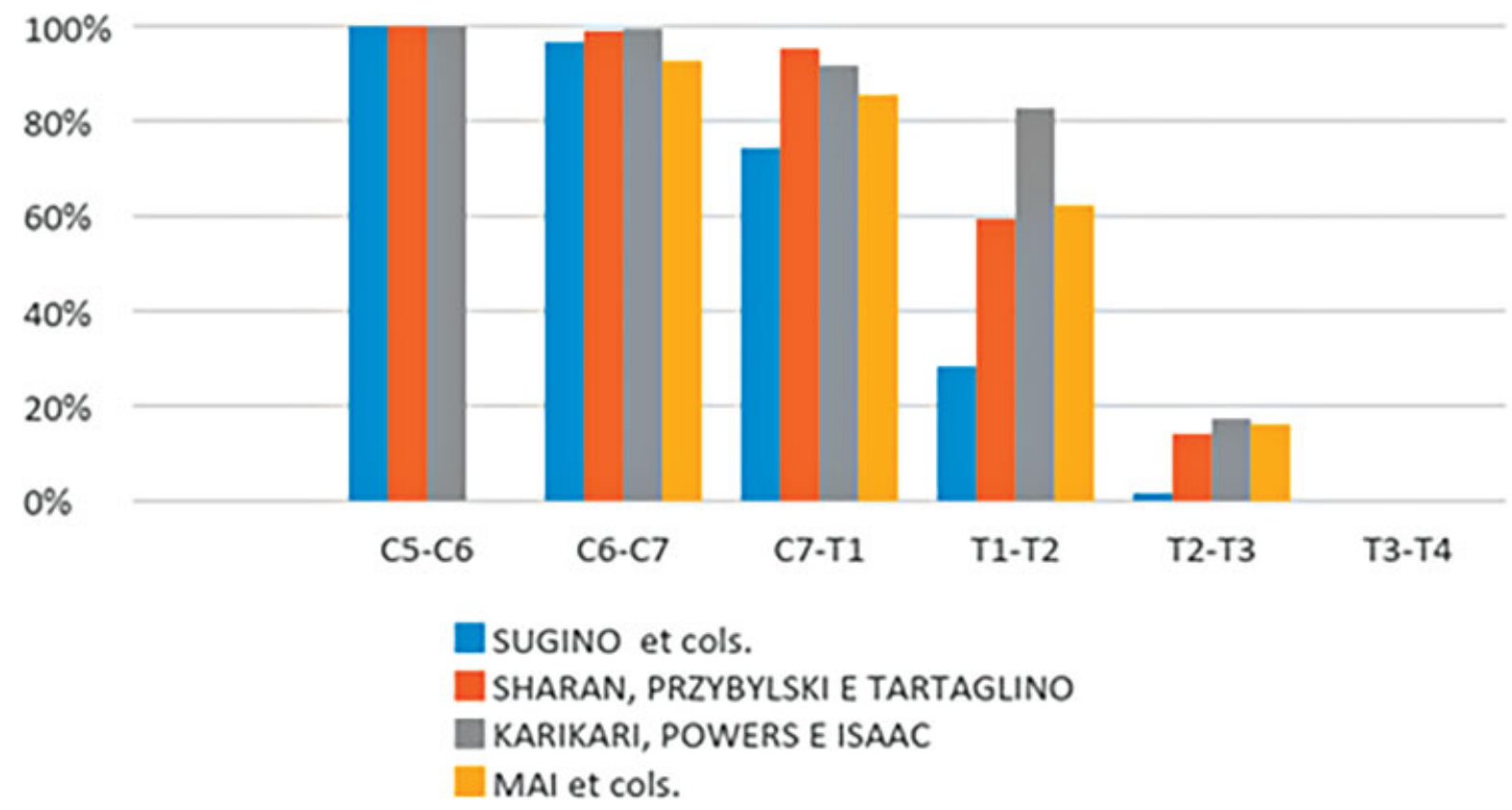

Fig. 7 Distribuição da linha do disco intervertebral (IDL) em diferentes estudos. 


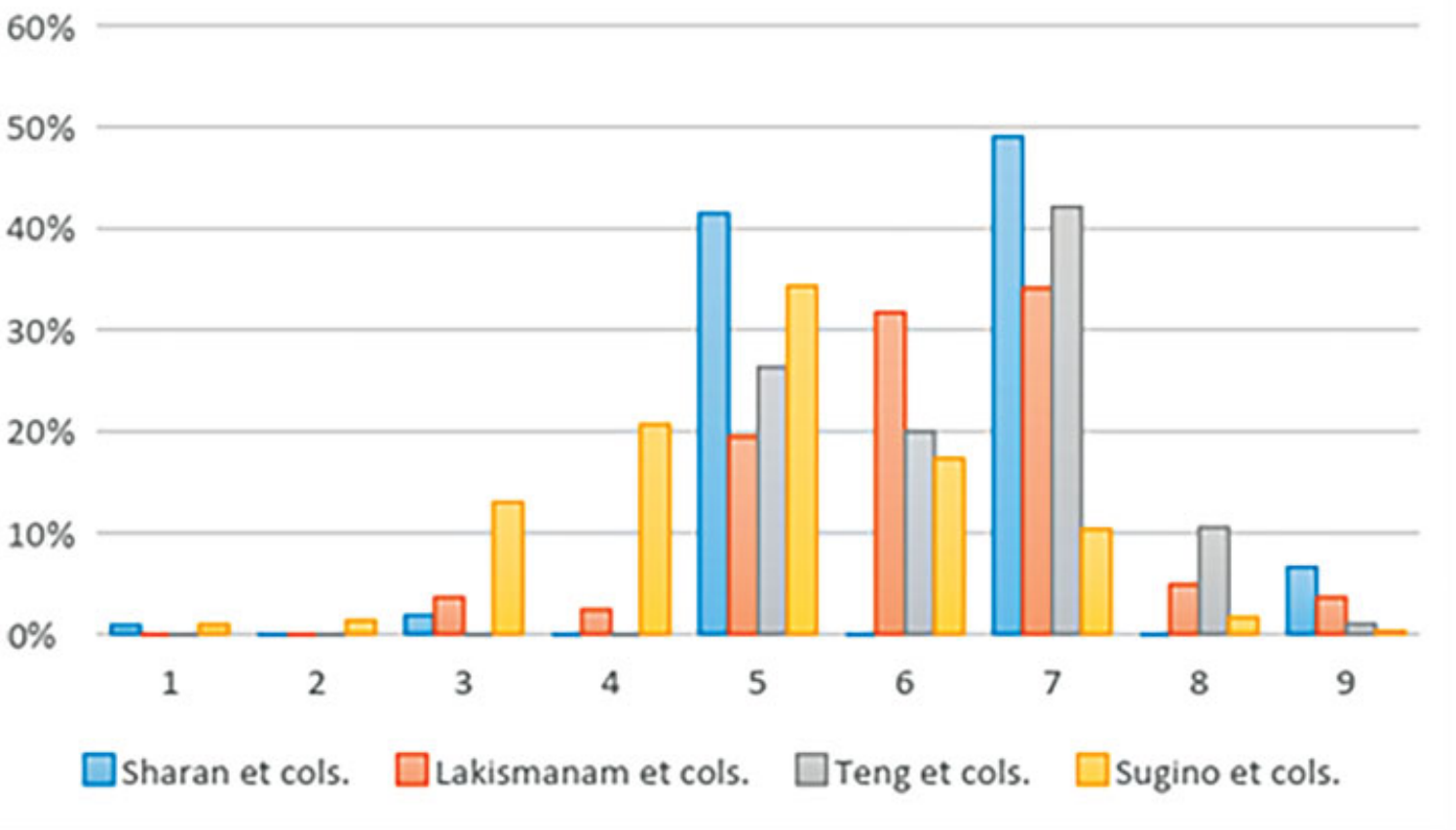

Fig. 8 Distribuição do nível horizontal acima do esterno (HLS) em diferentes estudos.

normalmente separados por transições suaves. Ambos os valores angulares foram maiores em homens e pacientes mais velhos. Acreditamos que esses achados sejam decorrentes de alterações cifóticas normais secundárias ao envelhecimento, em que há rotação dos níveis torácicos superiores e aumento da rotação angular. ${ }^{17}$

Ao analisar a IDL, não houve diferença entre os gêneros. No entanto, descobrimos que, com a idade, o acesso de níveis espinhais na junção cervicotorácica pode ser mais difícil por causa da migração cranial da linha do disco que passa acima da fúrcula esternal. Isso é uma consequência da rotação vertebral e não da translação vertical associada ao envelhecimento. Acreditamos também que, além da idade avançada, nossa população geográfica específica está mais exposta ao risco de osteotomia do esterno durante o acesso anterior da junção cervicotorácica. Este fato é uma observação anatômica que ocorre na população brasileira. $O$ aumento da inclinação de T1, indiretamente medido pela angulação do disco, é igualmente importante. Mais estudos são necessários para confirmar essa correlação em diferentes populações. Esse fenômeno é comum em pacientes mais velhos, como demonstrado por nosso estudo e pela literatura. O método que utilizamos para o planejamento cirúrgico é simples e confiável. Mesmo que o nível operatório não esteja acima do esterno, uma corpectomia pode ser feita no corpo vertebral acima do nível desejado para a realização da cirurgia, embora isso aumente a morbidade cirúrgica e altere totalmente o planejamento do procedimento.

Por ser um estudo observacional retrospectivo, há algumas limitações. Não pudemos testar nossa hipótese de forma prospectiva ou determinar a importância clínica de nosso estudo. Outros estudos já analisaram a capacidade de acesso da junção cervicotorácica por meio de TC. No entanto, nenhum estudo analisou os dados por comparação de diferentes faixas etárias e gênero. Mais dados de diferentes populações seriam necessários para melhor compreensão de nossos achados, mas a literatura não possui essas informações.

\section{Conclusão}

Em comparação a outras populações, nossa amostra apresentou mais casos de IDL e HLS cefálicos, indicando que menos níveis poderiam ser abordados anteriormente sem a necessidade de uma esternotomia. Ao comparar os grupos de diferentes gêneros, não houve diferença em IDL e HLS, enquanto VBA e IDLA tenderam a ser maiores em homens. O HLS foi semelhante entre as faixas etárias, sugerindo que não houve translação vertical entre o esterno e a coluna vertebral. Valores maiores de VBA e IDLA foram observados em grupos mais velhos em comparação aos mais jovens. A IDL ficou mais caudal em pacientes mais velhos devido à rotação vertebral ao invés de uma diferença translacional.

\section{Suporte Financeiro}

Esta pesquisa não recebeu nenhuma bolsa específica de agências de fomento nos setores públicos, comerciais ou sem fins lucrativos.

\section{Conflito de Interesses}

Possíveis conflitos de interesse: (R. L. S.) nada a declarar; (A. S. I.) nada a declarar; (S. C.) consultor Globus, em andamento, e consultor Zimmer Biomet, em andamento; (D. C.) nada a declarar; (L. M.) nada a declarar; (C. F. P. S. H.) nada a declarar. 


\section{Referências}

1 An HS, Vaccaro A, Cotler JM, Lin S. Spinal disorders at the cervicothoracic junction. Spine (Phila Pa 1976) 1994;19(22): 2557-2564

2 Huang YX, Tian NF, Chi YL, Wang S, Pan J, Xu HZ. Mini-open anterior approach to the cervicothoracic junction: a cadaveric study. Eur Spine J 2013;22(07):1533-1538

3 Fraser JF, Diwan AD, Peterson M, et al. Preoperative magnetic resonance imaging screening for a surgical decision regarding the approach for anterior spine fusion at the cervicothoracic junction. Spine (Phila Pa 1976) 2002;27(07):675-681

4 Huang YX, Ni WF, Wang S, et al. Anterior approaches to the cervicothoracic junction: a study on the surgical accessibility of three different corridors based on the CT images. Eur Spine J 2010; 19(11):1936-1941

5 Cho W, Buchowski JM, Park Y, Maeda T, Nabb CE, Riew KD. Surgical approach to the cervicothoracic junction: can a standard Smith-Robinson approach be utilized? J Spinal Disord Tech 2012; 25(05):264-267

6 Karikari IO, Powers CJ, Isaacs RE. Simple method for determining the need for sternotomy/manubriotomy with the anterior approach to the cervicothoracic junction. Neurosurgery 2009; 65(6, Suppl)E165-E166

7 Lakshmanan P, Lyons K, Davies PR, Howes JP, Ahuja S. Radiographic assessment of sternal notch level and its significance in approaching the upper thoracic spine. Am J Orthop 2009;38(04):E71-E74

8 Landes G, Harris PG, Sampalis JS, et al. Outcomes in the management of sternal dehiscence by plastic surgery: a ten-year review in one university center. Ann Plast Surg 2007;59(06):659-666

9 El Oakley RM, Wright JE. Postoperative mediastinitis: classification and management. Ann Thorac Surg 1996;61(03):1030-1036
10 Lesoin F, Thomas CE 3rd., Autricque A, Villette L, Jomin M. A transsternal biclavicular approach to the upper anterior thoracic spine. Surg Neurol 1986;26(03):253-256

11 Liu YL, Hao YJ, Li T, Song YM, Wang LM. Trans-upper-sternal approach to the cervicothoracic junction. Clin Orthop Relat Res 2009;467(08):2018-2024

12 Luk KD, Cheung KM, Leong JC. Anterior approach to the cervicothoracic junction by unilateral or bilateral manubriotomy. A report of five cases. J Bone Joint Surg Am 2002;84(06): 1013-1017

13 Falavigna A, Righesso O, Teles AR. Anterior approach to the cervicothoracic junction: proposed indication for manubriotomy based on preoperative computed tomography findings. J Neurosurg Spine 2011;15(01):38-47

14 Sharan AD, Przybylski GJ, Tartaglino L. Approaching the upper thoracic vertebrae without sternotomy or thoracotomy: a radiographic analysis with clinical application. Spine (Phila Pa 1976) 2000;25(08):910-916

15 Teng H, Hsiang J, Wu C, et al. Surgery in the cervicothoracic junction with an anterior low suprasternal approach alone or combined with manubriotomy and sternotomy: an approach selection method based on the cervicothoracic angle. J Neurosurg Spine 2009;10(06):531-542

16 Mai HT, Mitchell SM, Jenkins TJ, Savage JW, Patel AA, Hsu WK. Accessibility of the Cervicothoracic Junction Through an Anterior Approach: An MRI-based Algorithm. Spine (Phila Pa 1976) 2016; 41(01):69-73

17 Park HY, Lee SH, Park SJ, Kim ES, Lee CS, Eoh W. Surgical management with radiation therapy for metastatic spinal tumors located on cervicothoracic junction: a single center study. J Korean Neurosurg Soc 2015;57(01):42-49 